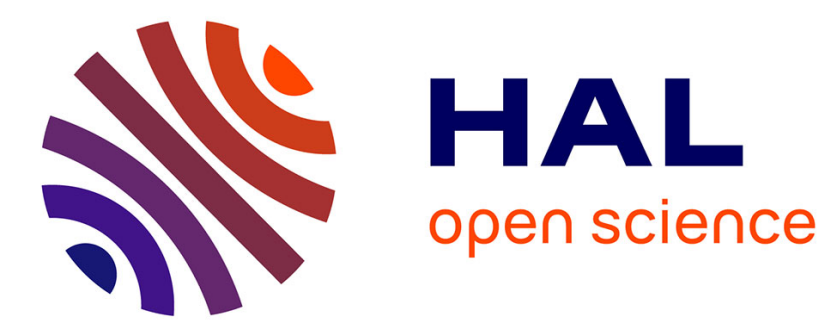

\title{
Turbulence driven widening of the near-SOL power width in ASDEX Upgrade H-Mode discharges
}

T. Eich, P. Manz, R.J. Goldston, Pascale Hennequin, P. David, M. Faitsch, B. Kurzan, B. Sieglin, E. Wolfrum

\section{- To cite this version:}

T. Eich, P. Manz, R.J. Goldston, Pascale Hennequin, P. David, et al.. Turbulence driven widening of the near-SOL power width in ASDEX Upgrade H-Mode discharges. Nuclear Fusion, 2020, 60 (5), pp.056016. 10.1088/1741-4326/ab7a66 . hal-02565895

\section{HAL Id: hal-02565895 \\ https://hal.science/hal-02565895}

Submitted on 16 Dec 2020

HAL is a multi-disciplinary open access archive for the deposit and dissemination of scientific research documents, whether they are published or not. The documents may come from teaching and research institutions in France or abroad, or from public or private research centers.
L'archive ouverte pluridisciplinaire HAL, est destinée au dépôt et à la diffusion de documents scientifiques de niveau recherche, publiés ou non, émanant des établissements d'enseignement et de recherche français ou étrangers, des laboratoires publics ou privés. 


\title{
Turbulence driven widening of the near-SOL power width in ASDEX Upgrade H-Mode discharges
}

\author{
T. Eich, ${ }^{1}$ P. Manz,${ }^{1}$ R.J. Goldston, ${ }^{2}$ P. Hennequin, ${ }^{3}$ P. David, ${ }^{1}$ M. Faitsch, ${ }^{1}$ B. Kurzan,${ }^{1}$ \\ B. Sieglin, ${ }^{1}$ E. Wolfrum,${ }^{1}$ the ASDEX Upgrade team,${ }^{4}$ and the EUROfusion MST1 team ${ }^{5}$ \\ ${ }^{1}$ Max-Planck-Institut für Plasmaphysik, \\ Boltzmannstraße 2, D-85748 Garching, Germany \\ ${ }^{2}$ Princeton Plasma Physics Laboratory, \\ Princeton, NJ08543, United States of America \\ ${ }^{3}$ LPP, Ecole Polytechnique, CNRS, 91128 Palaiseau, France \\ 4 see the author list of H.Meyer at al. 2019 Nucl. Fusion 59112014 \\ ${ }^{5}$ see the author list Labit, B. et al. 2019 Nucl. Fusion 59086020
}

(Dated: December 3, 2019) 


\begin{abstract}
Divertor heat flux data from infra red (IR) from various tokamaks in H-Mode regime show that the power width, $\lambda_{\mathrm{q}}$, scales approximately like $1.6(R / a) \rho_{s, p o l}$. However, the IR based scaling comes with the restriction that only low-gas-puff discharges were considered. Here we set up a new data base covering low edge density data but also including high density plasmas reaching the H-mode density limit. We use Thomson-scattering to measure the electron temperature decay length which will set the near-SOL power width through parallel heat conduction. We present scaling results for the density, temperature and pressure separatrix fall-off lengths. As the principal result we derive a generalized power width scaling which reads as $\lambda_{\mathrm{q}} \propto \rho_{\mathrm{s}, \mathrm{pol}} \cdot\left(1+3 \alpha_{\mathrm{t}}^{1.3}\right)$ where $\alpha_{\mathrm{t}}$ describes a normalized collisionality $\left(\alpha_{\mathrm{t}}=3 \cdot 10^{-18} R q^{2} n Z_{\mathrm{eff}} T^{-2}\right)$. The parameter $\alpha_{\mathrm{t}}$ describes the relative importance of the interchange effect on drift-wave turbulence as proposed by Scott and is found to be closely linked to the diamagnetic parameter $\alpha_{d}$ introduced by Rogers, Drake and Zeiler (RDZ). The edge operational space for ASDEX Upgrade displays a boundary qualitatively in agreement with the region labelled as density limit in the edge plasma phase space proposed by RDZ. The data base shows in the limit of low edge densities $\left(\alpha_{\mathrm{t}} \simeq 0.1\right)$ accurate agreement to the IR based power width scaling. At elevated separatrix densities $\left(\alpha_{\mathrm{t}} \simeq 1\right)$ the power width is broadened by a factor of up to about three accompanied by a reduction of the $\mathrm{H}_{98, y 2}$ confinement factor. Plasmas with higher triangularity show higher values for $\mathrm{H}_{98, y 2}$ in the same range of normalized collisionality.
\end{abstract}

PACS numbers: 52.55.Fa 


\section{INTRODUCTION}

Operation of tokamaks with H-Mode characteristics [1] at high densities and at least partially detached divertor conditions is generally foreseen for future high-power fusion systems [2], including ITER [3]. The ease of access to divertor detachment can only be assessed when the power width is known in conditions where the separatrix density is high [4-6]. A multi-machine study was carried out to compare measurements of divertor heat flux from various tokamaks (JET, DIII-D, ASDEX Upgrade, Alcator C-Mod, NSTX and MAST) finding that the power width $\lambda_{\mathrm{q}}$ for H-mode operation is inversely proportional to the poloidal magnetic field, $B_{p o l}$. Equally important, no dependence on the machine size was detected $[7,8]$. Both aspects can be interpreted as a combination of ion-carried neoclassical drift-orbit particle losses and anomalous electron heat diffusion filling that loss channel [9-13]. This prediction for the near SOL power width matches closely experimental data and is well described by

$$
\lambda_{\mathrm{q}} \simeq 1.6 \times \frac{a}{R} \rho_{s, p o l}, \quad \rho_{s, p o l}=\frac{\sqrt{m_{D} T_{s e p}}}{e B_{p o l}}
$$

with minor and major radii $a$ and $R, m_{D}$ the ion mass, $T_{\text {sep }}=T_{i}=T_{e}$ being the separatrix temperature of electrons and ions and $B_{p o l}$ the poloidally averaged poloidal magnetic field. This way the power width for ITER was predicted to be of the order of $1 \mathrm{~mm}$. All decay lengths in this paper are given as poloidally averaged values except where noted. Typically the poloidally averaged value is about 1.8 times larger than one would get for outer mid plane (OMP) mapping. This original scaling comes with the restriction that only low-gaspuff discharges were considered. The pressure fall-off length is calculated from the estimated density and temperature fall-off lengths, explicitly $\lambda_{\mathrm{p}}=\left(\lambda_{\mathrm{T}}^{-1}+\lambda_{\mathrm{n}}^{-1}\right)^{-1}$.

Here we set up a new data base containing data with low to highest separatrix density plasmas by deuterium gas puffing until reaching the H-mode density limit (see [14] and references within). For a few cases small or moderate amounts of nitrogen seeding is present. It is carefully checked that such impurity seeded discharges do not bias the regression results by including/excluding them. We use Thomson-scattering data to measure the electron density and temperature decay lengths at the separatrix. Experimental details of this method are described in detail in $[15,16]$. In the latter work by Sun et al. good agreement is reported for comparison of the power width scaling reconstructed by divertor measurements 
and the electron temperature decay length measured at the outer equatorial mid plane by the Thomson-scattering diagnostic. Explicitly the correlation in Eq. 2 due to the dominance of Spitzer-Härm conduction is verified, in line with direct comparisons on ASDEX Upgrade $[17]$ :

$$
\lambda_{\mathrm{T}} \simeq \frac{7}{2} \lambda_{\mathrm{q}}
$$

However, corrections due to kinetic effects to this simple assumption are reported e.g. from DIII-D $[18,19]$ but not addressed in this contribution.

For this reason either $\lambda_{\mathrm{T}}$ or $\lambda_{\mathrm{q}}$ are considered here as the power width in tokamaks as they both equally well describe the volume in which the power entering the SOL must be dissipated in order to meet the technical requirement for divertor integrity [3]. Taken together, this makes an extension of the former work possible by establishing a scaling for $\lambda_{T}$ measured at the outer mid plane and including plasmas with highest accessible edge densities given by the so called H-mode density limit [20].

This attempt is motivated by the experimental findings of Sun [15] that at elevated separatrix densities a widening of the power width for H-modes in AUG is observed outside the scaling prediction. Furthermore various theoretical contributions consider electron turbulence to become stronger at elevated edge pressure $[12,13,21-26]$. Possibly such elevated turbulence would lead to an increase of the power width with machine size and thus would cause a widening of the power width for larger machines than currently considered. The work by Chang [12] using the XGC1 code, for example, predicts a significant widening of the power width for ITER $(\mathrm{Q}=10)$ up to $\lambda_{\mathrm{q}} \approx 5 \mathrm{~mm}$ at the outer equatorial mid-plane whilst good agreement to the empirical scaling for operating devices is found. However, it should be noted that these predictions are not associated with enhanced collisionality but are reported to be subject to a change of the turbulence mechanism.

\section{THE EDGE PLASMA OPERATIONAL SPACE IN ASDEX UPGRADE}

In order to quantify the strength of anomalous fluxes we apply the turbulence control parameters, $\alpha_{\mathrm{MHD}}$ and $\alpha_{\mathrm{d}}$ from the work of Roger, Drake, Zeiler (RDZ) introducing the concept of an edge plasma operational space or plasma phase space as named in [27]. We compare to a similar approach by Scott $[28,29]$. We use these turbulence control parameters to identify 
TABLE I: Discharge parameters of analyzed H-Mode plasmas. The data base for L-Mode discharges are given for completeness.

the regions of enhanced turbulence that may effect the electron temperature, density and pressure decay widths. To justify our approach we present first a comparison of the edge plasma computational prediction as proposed by RDZ to a large data base for separatrix plasma conditions in ASDEX Upgrade. Already in 1999 Suttrop found agreement between experimental data and the RDZ proposed edge plasma prediction in ASDEX Upgrade [30]. Further agreement between experimental data and the simulation results by RDZ was also reported in the work by LaBombard [31] using probe measurements just outside the separatrix for Alcator C-Mod plasmas. A major advantage of the data base here presented in comparison to these earlier attempts $[30,31]$ is the use of a high resolution edge Thomson scattering diagnostic allowing to measure a large range of operational conditions in ASDEX Upgrade. This includes L-Mode and H-Mode plasmas including for both density limited discharges. Tab. I presents the discharge parameters used for the studies presented here. Overall 150 plasma discharges are analyzed, the great majority of them (130 discharges) in H-Mode. For each discharge typically 10-20 data points are generated.

\section{A. Definition of a turbulence parameter based on the RDZ/Scott parameter}

In this section we motivate a modified turbulence control parameter $\alpha_{\mathrm{t}}$ in order to use this value for regression studies on the SOL electron density, temperature and pressure decay lengths. Even though we are aware that plasma edge turbulence is a complex phenomenon that can hardly be described by two parameters only, to quantify a possible broadening of the near SOL width by turbulence, a control parameter of plasma edge turbulence is desirable. This control parameter should describe the strength of the plasma edge turbulence and its transport. In their fundamental work Rogers, Drake and Zeiler [27] proposed that the 
plasma edge is controlled by two main parameters, the ideal MHD ballooning parameter

$$
\alpha_{\mathrm{MHD}}=R \hat{q}_{\mathrm{cyl}}^{2} \frac{\beta}{\lambda_{\mathrm{p}}}
$$

where $\beta=4 \mu_{0} p_{\mathrm{e}} / B_{\mathrm{tot}}^{2}, \lambda_{\mathrm{p}}$ the plasma pressure gradient scale length, $B_{\text {tot }}$ the magnetic field strength and $R$ as the major radius. We use further $A=R / a$ for the aspect ratio and define with $\kappa_{\text {geo }}$ for the elongation and $\delta$ for the plasma triangularity at the separatrix

$$
\hat{\kappa}=\sqrt{\frac{1+\kappa_{\text {geo }}^{2}\left(1+2 \delta^{2}-1.2 \delta^{3}\right)}{2}}
$$

and with this we define

$$
\hat{q}_{\mathrm{cyl}}=\frac{B_{\mathrm{tor}}}{B_{\mathrm{pol}}} \cdot \frac{\hat{\kappa}}{A} \text { with } B_{\mathrm{pol}}=\frac{\mu_{0} I_{\mathrm{p}}}{2 \pi a \hat{\kappa}} .
$$

A turbulence control parameter, the so-called diamagnetic parameter $\alpha_{\mathrm{d}}$, controlling the impact of drift-wave dynamics on the interchange instability, is defined as

$$
\alpha_{\mathrm{d}}=\sqrt{\frac{\left(m_{\mathrm{i}} / m_{\mathrm{e}}\right) c_{\mathrm{s}}}{0.51 \nu_{\mathrm{ei}}\left(2 \pi \hat{q}_{\mathrm{cyl}}\right)^{2} R}}\left(\frac{R}{\lambda_{\mathrm{p}}}\right)^{1 / 4} .
$$

The electron ion collision frequency is denoted as $\nu_{\mathrm{ei}}$, ion and electron mass as $m_{\mathrm{i}, \mathrm{e}}$ and ion sound speed as $c_{\mathrm{s}}=\sqrt{T_{\mathrm{e}} / m_{\mathrm{i}}}$. The extended parallel scale length due to the elongation $\kappa_{g e o}$ when compared to the circular cross-section is taken into account in $\hat{q}_{c y l}$.

Scott $[28,29]$ investigated the same phenomenon from another perspective, namely the impact of the interchange effect on drift-wave turbulence controlled by the so-called resistive ballooning parameter $C \omega_{\mathrm{B}}$. Within the work of Scott a parameter is defined as

$$
\omega_{\mathrm{B}}=\frac{2 \lambda_{\mathrm{p}}}{R}\left(1+\frac{1}{\bar{Z}}\right)
$$

which sets the strength of the curvature drive and hence the strength of the interchange turbulence. The average ion charge enters here as $\bar{Z}=n_{e} /\left(\sum_{j} n_{j}\right)$. The thermal diffusivities $\chi_{\mathrm{e}, \mathrm{i}}$ approximately increase linearly with the normalised collisionality $\chi_{\mathrm{e}, \mathrm{i}} \sim C \chi_{\mathrm{GB}}$ with gyroBohm diffusivity $\chi_{\mathrm{GB}}[32]$. Hence, the transport is controlled in parts by $C$. The normalised collisionality is given by

$$
C=0.51 \nu_{\mathrm{ei}} \frac{\lambda_{\mathrm{p}}}{c_{\mathrm{s}}}\left(\frac{m_{e}}{M_{i}}\right)\left(\frac{\hat{\underline{q}}_{\mathrm{cyl}} R}{\lambda_{\mathrm{p}}}\right)^{2},
$$

where $\nu_{\mathrm{ei}}$ is the electron-ion Braginskii collision frequency, $\left(\hat{q}_{\mathrm{cyl}} R / \lambda_{\mathrm{p}}\right)^{2}$ the typical ratio of parallel to perpendicular scale length controlling the adiabaticity of the electrons and $\lambda_{\mathrm{p}} / c_{\mathrm{s}}$ 
due to the time normalization. Some first-order adjustments have to be made for impure plasmas which are discussed in the appendix. Similar to $\alpha_{\mathrm{d}}$ the parameter $C \omega_{\mathrm{B}}$ is found through simulations to control the relative strength of interchange and drift-wave turbulence by the cross-phase between pressure and potential perturbations. Whereas the transition to the ideal ballooning regime at a critical $\alpha_{\mathrm{MHD}}$ is rather sharp, the transition to interchange dominated turbulence (also called resistive ballooning turbulence) is gradual [32]. Both parameters, $C$ and $\omega_{\mathrm{B}}$, depend on the gradient length scale $\lambda_{\mathrm{p}}$, however, the combination $C \omega_{\mathrm{B}}$ does not depend on $\lambda_{\mathrm{p}}$.

In order to develop experimental scaling laws for decay length, we propose a turbulence parameter

$$
\alpha_{\mathrm{t}} \equiv C \omega_{\mathrm{B}}=1.02 \frac{\nu_{\mathrm{ei}}}{c_{\mathrm{s}}} \frac{m_{e}}{M_{i}} \hat{q}_{\mathrm{cyl}}^{2} R \cdot\left(1+\frac{1}{\bar{Z}}\right)
$$

The motivation of this new parameter is to avoid collinearity with the pressure decay length by itself so that it can be used for regression studies. Explicitly the turbulence parameter is written (see appendix for the influence of $Z_{\text {eff }}$ on $\nu_{e i}$ ) as:

$$
\alpha_{\mathrm{t}}=\hat{q}_{\mathrm{cyl}}^{2} R \cdot \sqrt{\frac{m_{e}}{\bar{M}}} \cdot \frac{1.02 \cdot \sqrt{2} e^{4} \ln \Lambda n_{\mathrm{e}}}{12 \pi^{3 / 2} \epsilon_{0}^{2} T_{\mathrm{e}}^{2}} \cdot \sqrt{\bar{Z}}(1+1 / \bar{Z}) \cdot Z_{\mathrm{eff}} f_{\mathrm{Z}_{\mathrm{eff}}} .
$$

The majority of our data base has little or no impurities so that a good approximation for our data base is given within an uncertainty of less than $10 \%$ by:

$$
\sqrt{\frac{m_{e}}{\bar{M}}} \sqrt{\bar{Z}} \cdot(1+1 / \bar{Z}) \cdot Z_{\mathrm{eff}} f_{\mathrm{Z}_{\mathrm{eff}}} \simeq 1.9 \sqrt{\frac{m_{\mathrm{e}}}{m_{\mathrm{D}}}} Z_{\mathrm{eff}} .
$$

We estimate the Coulomb logarithm $\ln \Lambda \approx 13.7$ by using the mean values for $T_{\text {sep }}=91 \mathrm{eV}$ and $n_{\text {sep }}=2.5 \cdot 10^{19} \mathrm{~m}^{-3}$ and rewrite Eq. 10 as

$$
\alpha_{\mathrm{t}} \approx 3 \cdot 10^{-18} \hat{q}_{\mathrm{cyl}}^{2} R \frac{n_{\mathrm{e}}}{T_{\mathrm{e}}^{2}} Z_{\mathrm{eff}} .
$$

For clarity we underline that the actual values for $\alpha_{\mathrm{t}}$ are calculated according to Eq. 10 . However, no changes w.r.t. content of this paper are notable when Eq. 12 is used.

We are interested to compare and validate the numerical values for $\alpha_{\mathrm{t}}$ and $\alpha_{\mathrm{d}}$. Balancing $\alpha_{\mathrm{d}}$ from Eq.(6) and $\alpha_{\mathrm{t}}$ from Eq.(9) yields (for $\bar{Z}=1$ )

$$
\alpha_{\mathrm{d}}=\frac{1}{\pi} \cdot\left(\frac{R}{\lambda_{\mathrm{p}}}\right)^{-\frac{1}{4}} \cdot \alpha_{\mathrm{t}}^{-\frac{1}{2}}
$$

In the work by RDZ the plasma phase space was derived for a fixed gradient scale length $\lambda_{\mathrm{p}} / R=0.005$, the latter value was chosen to match experimental conditions for ASDEX 
Upgrade operation in 1998 for discharges at 1 MA and 2 T. Our data base covers discharges for ASDEX Upgrade ranging from 0.6 MA to 1.2 MA, hence the gradient scale length chosen for the numerical simulations by RDZ are well in line with the observed experimental values $\lambda_{\mathrm{p}} / R=0.0027-0.027$ with a mean value of $\lambda_{\mathrm{p}} / R=0.0067$ in our data base. As a result we find as a good approximation for our data base

$$
\alpha_{\mathrm{d}}=(1.11 \pm 0.31) \cdot \alpha_{\mathrm{t}}^{-\frac{1}{2}}
$$

This means that both $\alpha_{\mathrm{d}}$ and $\alpha_{\mathrm{t}}^{-\frac{1}{2}}$ should give about the same values for the entire data base which is confirmed in Fig. 1 (bottom left). The dashed lines represent the mean value and the experimental range of $\lambda_{\mathrm{p}} / R$ from Eq. 14 .

Finally, we are interested to compare $\alpha_{\mathrm{t}}$ to a standard notation of the edge collisionality. Here we see that the turbulence parameter and the collisionality are closely linked by $\hat{q}_{\text {cyl }}$ :

$$
\alpha_{\mathrm{t}} \simeq \frac{1}{100} \cdot \hat{q}_{\mathrm{cyl}} \nu_{\mathrm{e}}^{*} \quad \text { with } \quad \nu_{\mathrm{e}}^{*}=\frac{\pi \hat{q}_{\mathrm{cyl}} R}{1.03 \cdot 10^{16}} \frac{n_{\mathrm{e}}}{T_{\mathrm{e}}^{2}} Z_{\mathrm{eff}}
$$

\section{B. Comparison of the plasma phase space to the data base}

Figure 1 shows the plasma phase space using $\alpha_{\mathrm{MHD}}$ and $\alpha_{\mathrm{d}}$ on the l.h.s. and $\alpha_{\mathrm{t}}$ on the r.h.s. based on 150 discharges (2355 data points) in ASDEX Upgrade covering various operation conditions $\left(I_{\mathrm{p}}=0.6-1.2 \mathrm{MA}, B_{\text {tor }}=1.5-2.5 \mathrm{~T}, P_{\text {heat }}=0.3-20 \mathrm{MW}\right)$. As explained above these parameters are closely related and hence the figures look similar. Most of the plasma edge operational space is in the drift-wave dominated regime $\left(\alpha_{\mathrm{d}}>1, \alpha_{\mathrm{t}}<1\right)$, in particular all of the $\mathrm{H}$-mode discharges are in this regime. According to both [27, 28], interchange becomes dominant for $\left(\alpha_{\mathrm{d}}<1, \alpha_{\mathrm{t}}>1\right)$. This region displays only L-modes approaching the L-mode density limit. Roughly, the H-Mode density limit appears in a region $\left(\alpha_{\mathrm{d}} \simeq 1, \alpha_{\mathrm{t}} \simeq 1\right)$ where both mechanisms are equally important.

The turbulence parameter $\alpha_{\mathrm{t}}$ in conjunction with $\alpha_{\mathrm{MHD}}$ describes the edge plasma phase space according to RDZ. The operationally inaccessible region at low $\alpha_{\mathrm{d}}$ (or high $\alpha_{\mathrm{t}}$ ) is clearly reproduced and the density limit leads to a steep boundary to the inaccessible region, also varying with $\alpha_{\mathrm{MHD}}$. Data where the discharges in L- and H-Mode are right at the density limit are labelled as L-Mode DL and H-Mode DL, respectively. It is interesting to note that the inaccessible region appears around $\alpha_{\mathrm{t}} \equiv C \omega_{\mathrm{B}} \simeq 0.5-1$ depending on $\alpha_{M H D}$. At $\alpha_{\mathrm{t}} \equiv C \omega_{\mathrm{B}} \simeq 1$ the transition from drift-wave to interchange dominated turbulence 

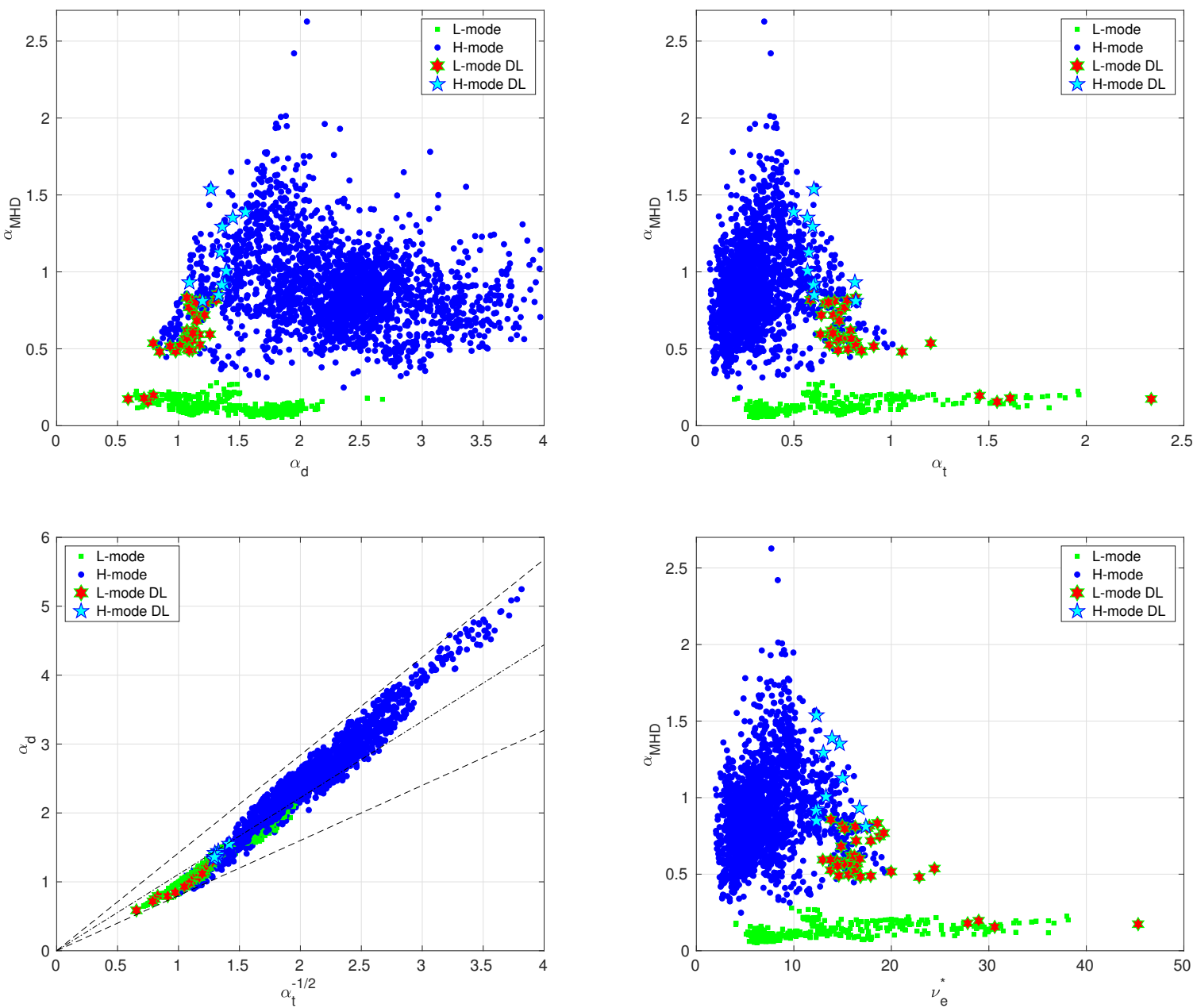

FIG. 1: A high level of similarity between two representations of the edge operational space is given. (top left) The plasma edge operational space for ASDEX Upgrade as proposed by RDZ using $\alpha_{\mathrm{MHD}}$ and $\alpha_{\mathrm{d}}$. The region of inaccessible operation is reproduced when $\alpha_{d}$ gets small. (top right) The plasma edge operational space by using $\alpha_{\mathrm{MHD}}$ and $\alpha_{\mathrm{t}}$. H-Modes are observed only for $\alpha_{\mathrm{t}}$ values below one. (bottom left) Comparison of $\alpha_{\mathrm{d}}$ and $\alpha_{\mathrm{t}}$. The dashed lines represent the mean and upper/lower boundary from Eq. 14. (bottom right) The simple electron collisionality orders the operational equally well as $\alpha_{\mathrm{t}}$.

is predicted by Scott [28]. Here the adiabatic response is reduced, which is in line with recent work [33, 34] explaining potentially the density limit as a consequence of decreased adiabaticity. At this boundary interchange-like transport becomes large as it is no longer stabilized by drift-wave modes. For L-mode plasmas $\alpha_{\mathrm{t}}$ ranges to about two. Plasmas with very high seeding levels are not included in this data base and hence radiation induced L- 
Mode disruptions are not included. Finally we note that no data are found above the ideal MHD limit at about $\alpha_{\mathrm{MHD}} \simeq 2-2.5$ consistent with the findings in [16]. In that earlier publication we stated that the H-Mode density limit is found at the boundary of ideal-MHD, hence a horizontal line in the edge plasma operational diagram at $\alpha_{M H D} \simeq 2-2.5$. This statement needs to be refined. The maximum achievable density at the separatrix without confinement degradation does appear to be given by the ideal ballooning limit. However, the final transition back to L-mode conditions appears when approaching a second operational boundary as attempts to raise the density further result in a reduction of $\alpha_{M H D}$ due to a strong relaxation of the plasma pressure gradients in the vicinity of the separatrix. This increase of the pressure decay length will be explicitly shown in section IV.

\section{PLASMA PARAMETER, PROFILE EVOLUTION AND FLUCTUATION MEASUREMENTS OF A SELECTED H-MODE DENSITY LIMIT DISCHARGE}

\section{A. Evolution of selected H-mode discharges of various plasma parameters}

We present a selected example of an H-Mode density limit discharge for which fluctuation measurements are available. In Fig. 2 the temporal evolution of the main discharge parameters are displayed. The discharge is chosen as it presents a large scan in density and the turbulence parameter reaches values of $\alpha_{\mathrm{t}} \simeq 1$. The plasma transits to H-Mode confinement conditions at about 2 seconds when the NBI heating is applied, at 3 seconds nitrogen seeding is applied. It is moderately heated by about $8 \mathrm{MW}$ heating power and reaches the H-Mode density limit due to combined deuterium and nitrogen puffing at about 4.1 seconds. From 4.1 to 4.7 seconds the discharge remains in L-Mode. The L-Mode phase eventually develops a Marfe and is terminated by a machine protection procedure. The marked time intervals correspond to (a) a type-I ELMy H-mode with $\mathrm{H}_{98, y 2} \simeq 1$, (b) reduced ELM amplitudes and degraded confinement while an increased Greenwald density fraction is observed. Then at about 3.6 seconds (c) further confinement degradation is present and finally (d) L-Mode characteristics are observed. From (a)-(d) both the heating power and radiation level stay roughly constant.

The evolution of the edge profiles measured by Thomson-scattering are displayed in Fig. 3. Additionally to the four chosen time points (a)-(d) which define the phases for which fluctua- 
tion measurements are available, two more profiles are presented. At 1.7152 seconds L-Mode profiles are displayed and at 2.2891 seconds the profiles during the early type-I ELMy phase are shown. The time windows used for averaging the profile data are in all 6 cases about $300 \mathrm{~ms}$. The L-Mode phase is characterized by a low separatrix temperature of $38 \mathrm{eV}$ and low density of $1.1 \cdot 10^{19} \mathrm{~m}^{-3}$. The electron temperature and density lengths are $\lambda_{\mathrm{T}}=31 \mathrm{~mm}$ and $\lambda_{\mathrm{n}}=54 \mathrm{~mm}$, respectively. After transition to H-Mode conditions, increases of the separatrix temperature to $92 \mathrm{eV}$ and density to $2.4 \cdot 10^{19} \mathrm{~m}^{-3}$ are observed while the decay lengths drop to less than half the L-Mode value, $\lambda_{\mathrm{T}}=13 \mathrm{~mm}$ and $\lambda_{\mathrm{n}}=21 \mathrm{~mm}$, respectively. While the separatrix density continuously increases due to the applied gas puffing rate, the electron temperature only reduces very little within measurement uncertainties. The estimated decay lengths for both electron temperature and density continuously increase. After the L-H back transition at 4.1 seconds, finally from the fall-off length at 4.5848 seconds are close to the values in the initial L-Mode phase at 1.7152 seconds $\left(\lambda_{\mathrm{T}}=29 \mathrm{~mm}\right.$ and $\left.\lambda_{\mathrm{n}}=57 \mathrm{~mm}\right)$ albeit with a twice as high electron temperature and about four times higher electron density. The turbulence parameter, thus, is identical within measurement uncertainties.

The observation of increased separatrix/SOL decay lengths in H-Mode at increasing Greenwald density fractions have been made much earlier and are furthermore also discussed in the context of increased radial transport as the cause for the density limit, see e.g. the work by LaBombard for C-Mod [35] and by Antar [36] for MAST, and references there in. We do not aim here to discuss the actual conditions for the back transition to L-Mode but are solely interested in the role of turbulence for widening the SOL decay lengths, i.e. combining empirically turbulence and drift-orbits is attempted in section IV.

\section{B. Fluctuation Measurements for \#34973}

Figure 4 compares the fluctuation time traces of $1.5 \mathrm{~ms}$ length measured around the four time points (a)-(d) described above. The fluctuation signal is measured by Doppler Back-Scattering (DBS), in the in-between phase of type-I ELMs. The DBS system [37] was configured in this case to cover the edge and SOL using both $\mathrm{V}$ and $\mathrm{W}$ bands in $\mathrm{X}$ mode. The trace shown corresponds to a channel probing the plasma at $\rho \simeq 0.995$ inside of the separatrix, with a perpendicular wave number in the mid-range $k \simeq 7-9 \mathrm{~cm}^{-1}$ corresponding to $k \rho_{s} \simeq 1$. Line averaged and separatrix densities continuously increase 


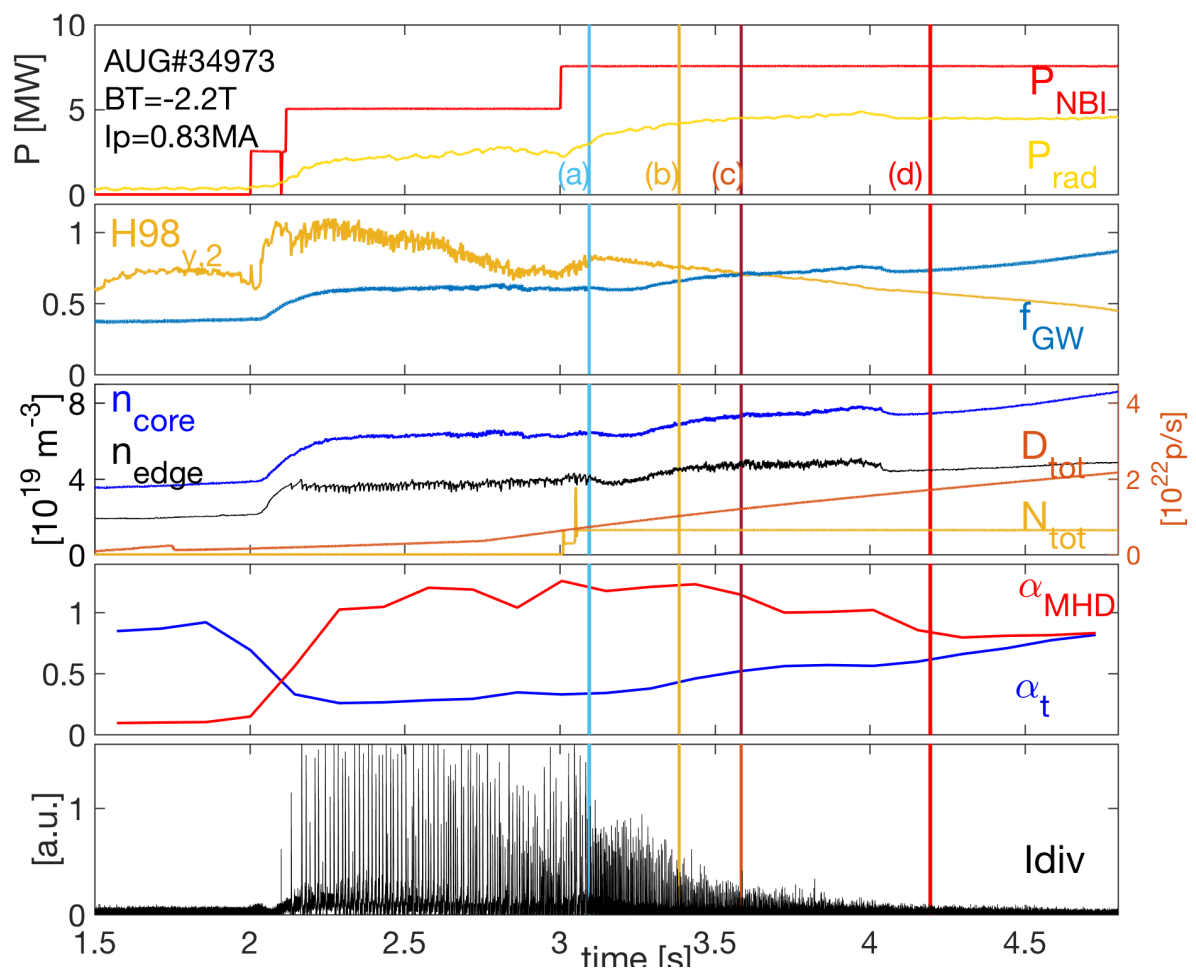

FIG. 2: Evolution of various discharge parameters for pulse \#34973. The presented signal (top to bottom) are neutral beam heating $\left(\mathrm{P}_{N B I}\right)$, radiation level $\left(\mathrm{P}_{r a d}\right)$ confinement factor and line averaged Greenwald density fraction $\left(\mathrm{f}_{G W}\right)$, density measurements from core and peripheral interferometer channels, $\alpha_{\mathrm{t}}$ and $\alpha_{M H D}$ evolution, and the divertor target shunt current $\left(\mathrm{I}_{\text {Div }}\right)$ measurements providing an ELM heat flux indicator. This discharge is a H-Mode with moderate heating which is undergoing an H-Mode density limit due to gas puffing at 4.1 seconds and remains in L-Mode later. The L-Mode phase eventually develops a Marfe and is terminated by machine protection. The marked time intervals correspond to (a) type-I ELMy H-mode with $\mathrm{H}_{98, y 2} \simeq 1$, (b) degraded confinement at increased $\mathrm{f}_{G W}$, (c) further degradation and (d) L-Mode characteristics.

during the discharge accompanied by a moderate reduction of the separatrix temperature. The elevating separatrix density corresponds to an increase in $\alpha_{\mathrm{t}}$ :

(a) at low $\alpha_{\mathrm{t}} \simeq 0.3$, in-between type-I ELMs, the fluctuation level is low except rare and short bursts (10 to $20 \mu \mathrm{s}$ ) which have a filament like structure and probably an electromagnetic character, as could be expected in H-Mode at high $\alpha_{\mathrm{MHD}}$; (b) at $\alpha_{\mathrm{t}} \simeq 0.5$, the fluctuation level is much stronger, dominated by the intense and repetitive bursts, typical of the inter ELMs phases in high density regimes [38]; broadband and incoherent fluctuations 

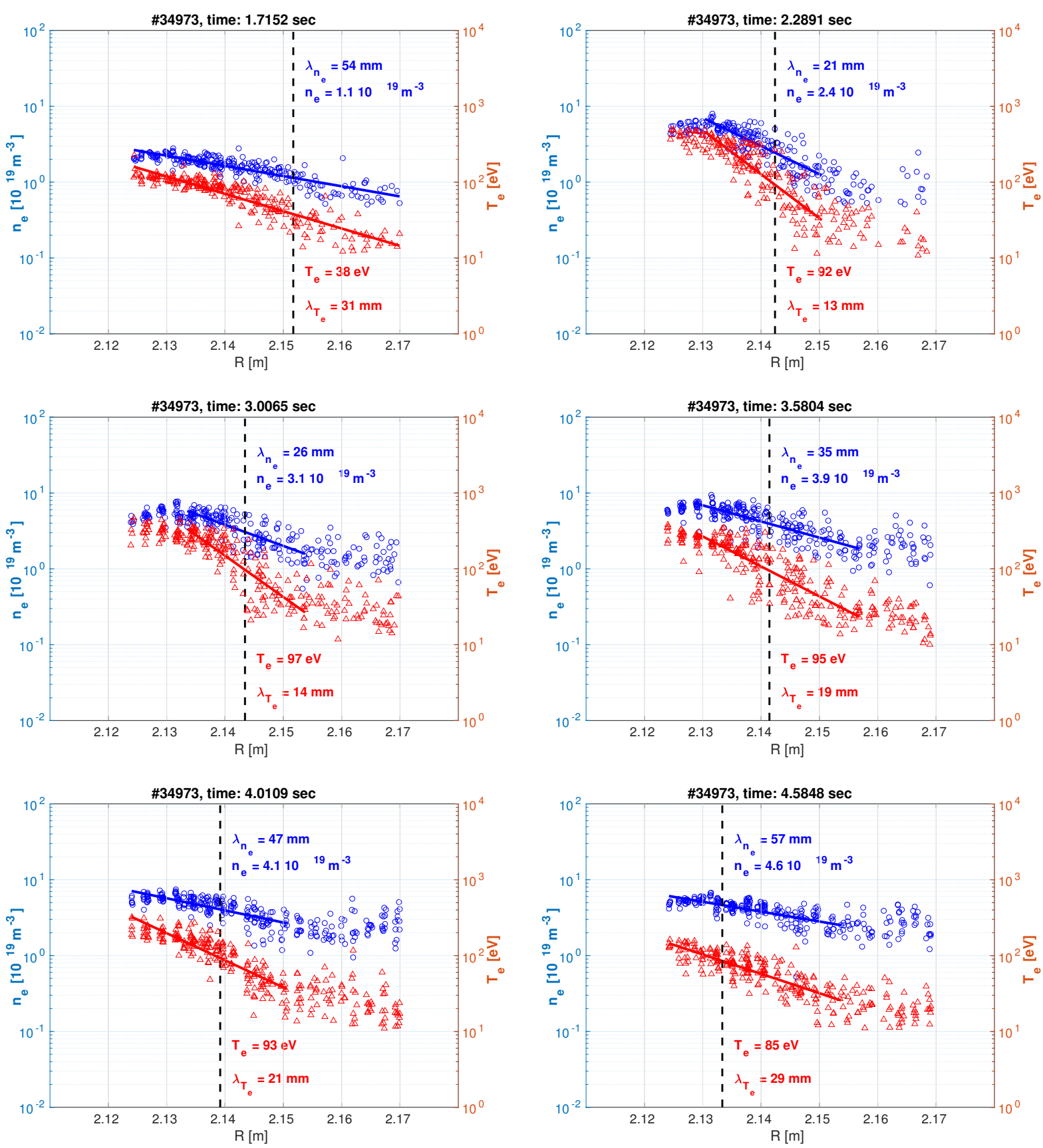

FIG. 3: Evolution of the electron temperature and density profile around the separatrix for discharge \# 34973. A significant widening of the density and temperature fall-off lengths is visible during the discharge evolution. Though the scatter of the data is high, it becomes clear that with increasing density and thus $\alpha_{\mathrm{t}}$ both density and temperature decay lengths are widening, however with a much larger effect on the density fall-off length.

also superimpose in (c). The latter dominate the signal in (d) after the back transition $\mathrm{H}-\mathrm{L}$ 


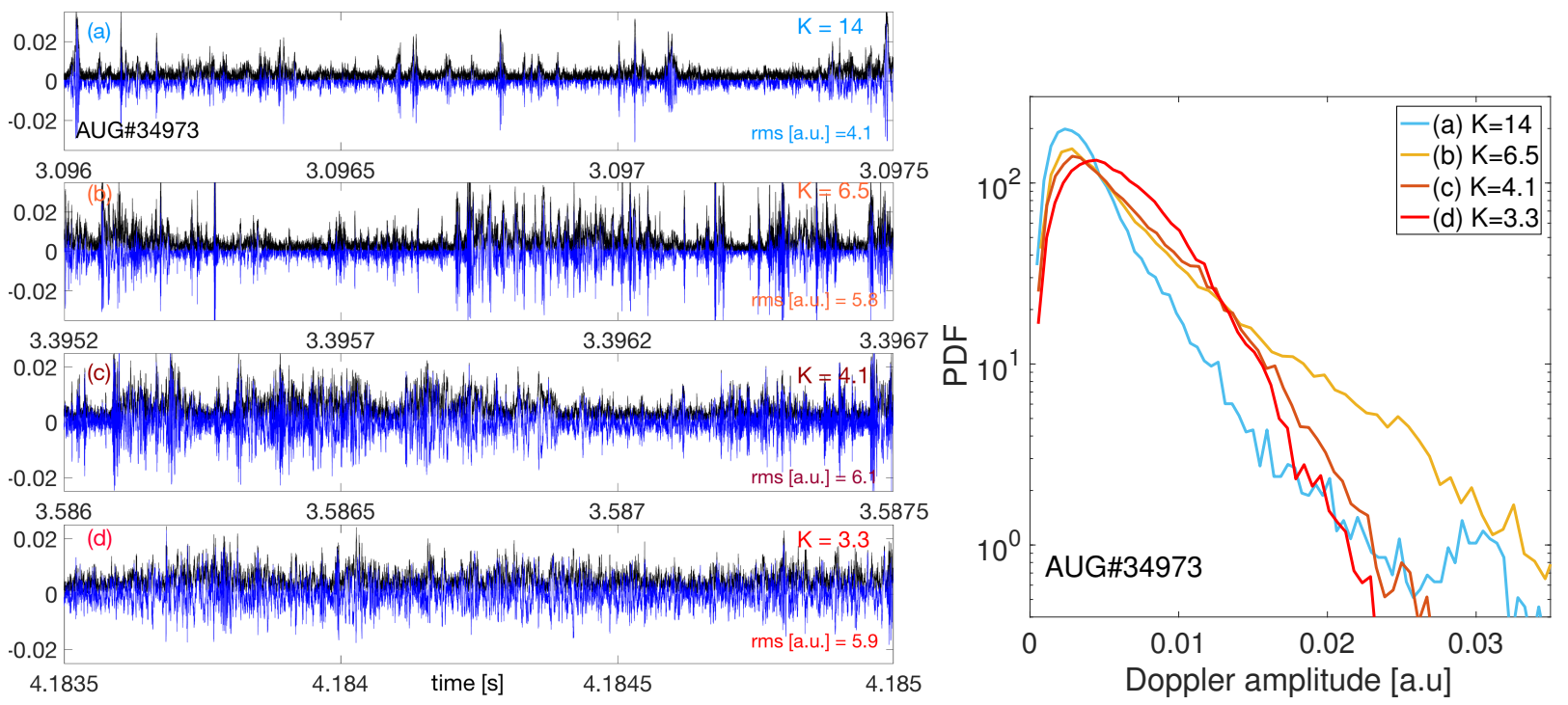

FIG. 4: Fluctuation measurements from Doppler Reflectometry for discharge \#34973 (left) plotted versus time for periods indicated in Fig. 2 and (right) the corresponding probability distribution functions for the time intervals.

( $\left.\alpha_{\mathrm{t}} \simeq 0.7\right)$; the overall level is high but the repetitive filaments are not visible anymore. The change in the dynamics from intermittent strong bursts to incoherent fluctuations is also well characterized by the Probability Distribution Function of the signal, shown in Fig. 4 (right): it strongly departs from log-normal distribution in case (a) and (b), which can be quantified by a high 4th moment (Kurtosis value $\mathrm{K}$ ) while $\mathrm{K}$ falls back to around 3 in (d), typical of L-Mode values.

In Fig. 5 the evolution of the fluctuation level versus $\alpha_{t}$ is shown for four discharges scanning from low H-Mode density until the HDL is hit. The turbulence intensity sharply increases with $\alpha_{t}$, consistently with a strong increase of transport. A saturation is observed at the highest $\alpha_{t}$. In general the turbulence level is not expected to be directly linear to the turbulence parameter, which is a limit between different turbulence drive. However, here the observed saturation is assumed to be at least partly due to measurement limitations. At large $\alpha_{\mathrm{t}}$, the observed change in the density gradient close to the separatrix might affect the DBS sensitivity (measurement volume) and wave number range. More importantly, at high turbulence level, exceeding a few percent, the DBS response can be non linear and saturate [39]. This effect should be negligible for $\alpha_{\mathrm{t}}$ typically less than 0.5: the turbulence is still very low at the edge barrier in the inter ELM phase, as can be seen in the DBS signal which 

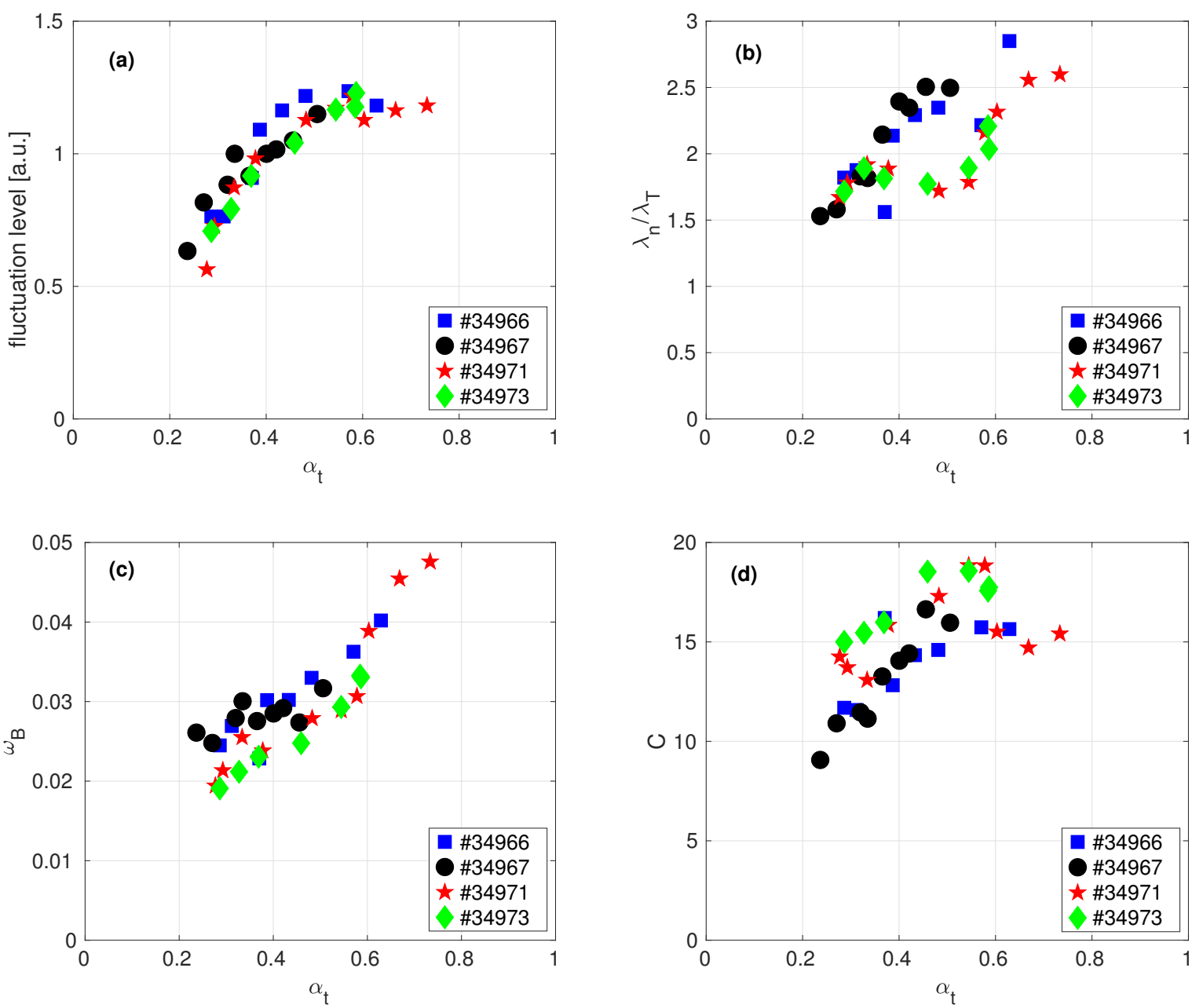

FIG. 5: (a) The fluctuation levels measured by Doppler Reflectometry versus the turbulence parameter $\alpha_{\mathrm{t}}$. A clear correlation is observed for four discharges for which DBS data are available. Discharges \# 34966 and \# 34967 are gas puffed only, whereas in discharges \# 34971 and \#34973 an additional nitrogen puff was applied. (b) The ratio between the density and temperature decay length increases with $\alpha_{\mathrm{t}}$. (c) The curvature drive $\left(\omega_{\mathrm{B}}\right)$ and $(\mathrm{d})$ normalized collisionality $(C)$ vs $\alpha_{\mathrm{t}}$. It can be seen that the increase of turbulence parameter $\alpha_{\mathrm{t}}$ is due to an increase of both the curvature drive and the normalized collisionality.

remains close to the noise level except for some rare bursts (Fig. 4 (a) upper panels); for $\alpha_{\mathrm{t}}$ around or larger than 0.5 a saturation gradually takes place as filaments become frequent and incoherent fluctuations overtake the signal (Fig. 4 (a) lower panels). 

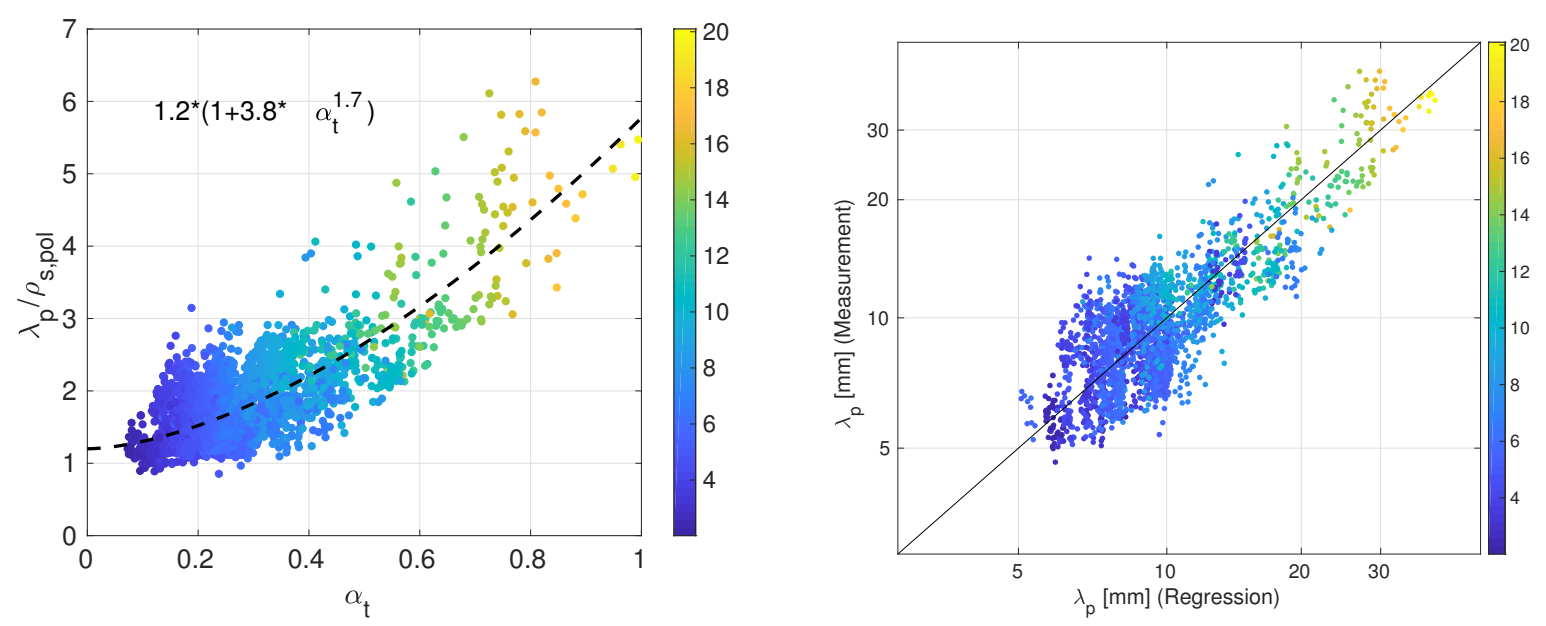

FIG. 6: (Left) Experimental values for the pressure fall-off length $\left(\lambda_{\mathrm{p}}\right)$ normalized by $\rho_{\mathrm{s}, \text { pol }}$ versus the turbulence parameter $\alpha_{\mathrm{t}}$. At low values of $\alpha_{\mathrm{t}} \simeq 0.1-0.2$ values around unity are found whereas a clear widening is observed when the turbulence parameter increases. (Right) Measured versus regression values of $\lambda_{\mathrm{p}}$ displayed in Eq.(18). Color coding represents the separatrix collisionality (defined in Eq. (15))

\section{GENERALIZED SOL DECAY LENGTH AND POWER WIDTH SCALING}

The new data base covers tokamak operation in ASDEX Upgrade from very low to highest edge densities achievable by gas puffing, mainly through the divertor gas inlet system. A minor number of discharges includes impurity seeding or phases with impurity seeding. The left plot in Fig. 6 displays the measured values of $\lambda_{\mathrm{p}} / \rho_{\mathrm{s} \text {,pol }}$ versus the turbulence parameter $\alpha_{\mathrm{t}}$. At low values for $\alpha_{\mathrm{t}} \simeq 0$ values around unity are found whereas a clear reduction in the pressure gradient is observed when the turbulence parameter increases. In order to find a regression law we propose an ansatz to describe the pressure decay length as a combination of the well established neoclassical drift-orbit like scaling following solely $\rho_{s, p o l}$ and the turbulence parameter $\alpha_{\mathrm{t}}$. We propose:

$$
\lambda_{\mathrm{p}}=\left(1+C_{\alpha} \alpha_{\mathrm{t}}^{\mathrm{a}}\right) C_{\rho} \rho_{\mathrm{s}, \mathrm{pol}}^{\mathrm{r}} .
$$

This compact expression allows for both the observed ordering of low densities with solely $\lambda_{\mathrm{p}} \propto \rho_{\mathrm{s}, \mathrm{pol}}$ as they were found in the multi-machine scaling but also its widening due to the anomalous electron heat transport controlled by $\alpha_{\mathrm{t}}$ as found in $[12,13]$. Seeded discharges are treated separately for the regression studies. We first exclude the seeded discharge 
because there is a large uncertainty on the values for $Z_{\text {eff }}$ at the separatrix position.

For the pressure fall-off length (both $\lambda_{\mathrm{p}}$ and $\rho_{\mathrm{s}, \text { pol }}$ in units of $\left.[\mathrm{mm}]\right)$ we find $\left(R^{2}=0.82\right.$, RMS: $2.3 \mathrm{~mm}, 1715$ data points used)

$$
\lambda_{\mathrm{p}}[\mathrm{mm}]=\left(1+(4.1 \pm 0.29) \alpha_{\mathrm{t}}^{1.6 \pm 0.11}\right) \cdot(1.3 \pm 0.16) \rho_{\mathrm{s}, \mathrm{pol}}^{0.95 \pm 0.07} .
$$

Here it is notable that the coefficient from the regression for the $\rho_{\mathrm{s} \text {,pol }}$ is close to unity $(\mathrm{r}=0.95 \pm 0.07)$. In order to employ a dimensional correct regression we set $r=1$ and find an equivalent regression law presented in Fig. 6 (right).

$$
\frac{\lambda_{\mathrm{p}}}{\rho_{\mathrm{s}, \mathrm{pol}}}=\left(1+(3.8 \pm 0.24) \alpha_{\mathrm{t}}^{1.7 \pm 0.12}\right) \cdot(1.2 \pm 0.07) \text {. }
$$

When including also the nitrogen seeded plasmas for the subset of the dedicated H-Mode density limit studies, the same regression values are found within fitting uncertainties albeit with a somewhat lower regression quality $\left(R^{2}=0.77\right.$, RMS: $2.3 \mathrm{~mm}, 2355$ data points used).

We extend this approach for the unseeded data to the density and temperature fall-off lengths. Here, again by least square-fitting for the identical data base, for the density fall-off length $\left(R^{2}=0.79\right.$, RMS: $\left.10.7 \mathrm{~mm}\right)$

$$
\lambda_{\mathrm{n}}[\mathrm{mm}]=\left(1+(9.5 \pm 0.8) \alpha_{\mathrm{t}}^{2.3 \pm 0.4}\right) \cdot(3.3 \pm 0.65) \rho_{\mathrm{s}, \mathrm{pol}}^{0.95 \pm 0.012}
$$

is obtained. We note that the exponent of $\rho_{\mathrm{s}, \mathrm{pol}}$ is also about one. The dimensional correct regression gives:

$$
\frac{\lambda_{\mathrm{n}}}{\rho_{\mathrm{s}, \mathrm{pol}}}=\left(1+(9.3 \pm 2.4) \alpha_{\mathrm{t}}^{2.3}\right) \cdot(3.1 \pm 0.75)
$$

In identical manner we obtain for the temperature fall-off length $\left(R^{2}=0.74\right.$, RMS: $3.4 \mathrm{~mm})$

$$
\lambda_{\mathrm{T}}[\mathrm{mm}]=\left(1+(3.1 \pm 0.28) \alpha_{\mathrm{t}}^{1.4 \pm 0.14}\right) \cdot(2.2 \pm 0.3) \rho_{\mathrm{s}, \mathrm{pol}}^{0.89 \pm 0.07}
$$

and dimensional correct:

$$
\frac{\lambda_{\mathrm{T}}}{\rho_{\mathrm{s}, \mathrm{pol}}}=\left(1+(3 \pm 0.5) \alpha_{\mathrm{t}}^{1.3 \pm 0.14}\right) \cdot(1.8 \pm 0.28)
$$

in good agreement, at low $\alpha_{\mathrm{t}}$, with Eq. $1 \& 2$. We detect this way that the influence of the turbulence parameter is much stronger on the density than on the temperature decay length, 

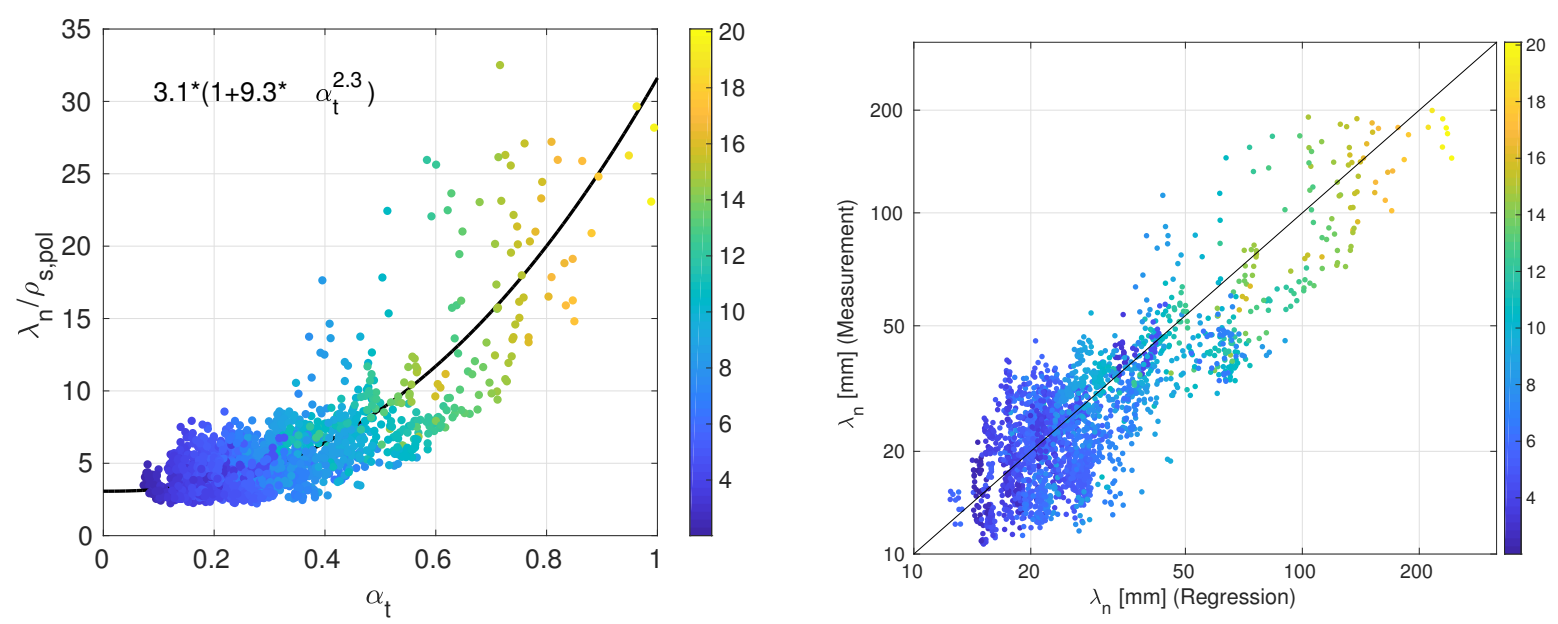

FIG. 7: (Left) Density decay length vs $\alpha_{\mathrm{t}}$. The dotted line show the turbulent broadening term of Eq. 20. (Right) Measurements versus regression values from Eq. 20. Color coding represents the separatrix collisionality (see Eq. 15)

both in the exponent and in the pre-factor. The ratio $\eta_{\lambda}=\lambda_{\mathrm{n}} / \lambda_{\mathrm{T}}$ consequently depends on $\alpha_{\mathrm{t}}$. In the limit $\alpha_{\mathrm{t}} \rightarrow 0$ a value for $\eta_{\lambda} \simeq 1.5$ is found and for $\alpha_{\mathrm{t}} \rightarrow 1$ we estimate $\eta_{\lambda} \simeq 4.5$ and hence $\eta_{\lambda}$ varies about a factor of three. This means when approaching high edge densities in H-mode plasmas, the density decay length widens far more strongly than the temperature decay length (as also presented in Fig. 3). Further profiles are presented in [41]. Figures 7 and 8 plot the measured versus the scaled values of the density and temperature decay lengths, respectively. Clearly a scatter remains. However, the fitting quality is similar to previous multi-machine scaling [8] while covering an operational range from low density towards high density limited discharges in a single machine.

We compare the new results to the divertor heat load based multi-machine power width scaling and high field results in Alcator C-Mod by Brunner[42]. The power width data base of Alcator C-Mod has been extended up to outer mid plane (MP) poloidal magnetic fields of $B_{\mathrm{pol}, \mathrm{MP}}>1.2 \mathrm{~T}$, hence ITER like values, for low edge density discharges suitable for such divertor heat load studies [42]. They report $\lambda_{q}[\mathrm{~mm}]=0.76 B_{p o l, M P}^{-0.96}$ and present a close match in absolute numbers to the multi-machine scaling which is (see [8], Reg.\#4) $\lambda_{q}[\mathrm{~mm}]=0.65 B_{p o l, M P}^{-1.11}$. Both attempts used the poloidal magnetic field and decay length at the outer mid plane and were applied to plasmas with best conditions suitable for divertor heat load studies namely high heating power and low gas puffing rates (thus low separatrix 

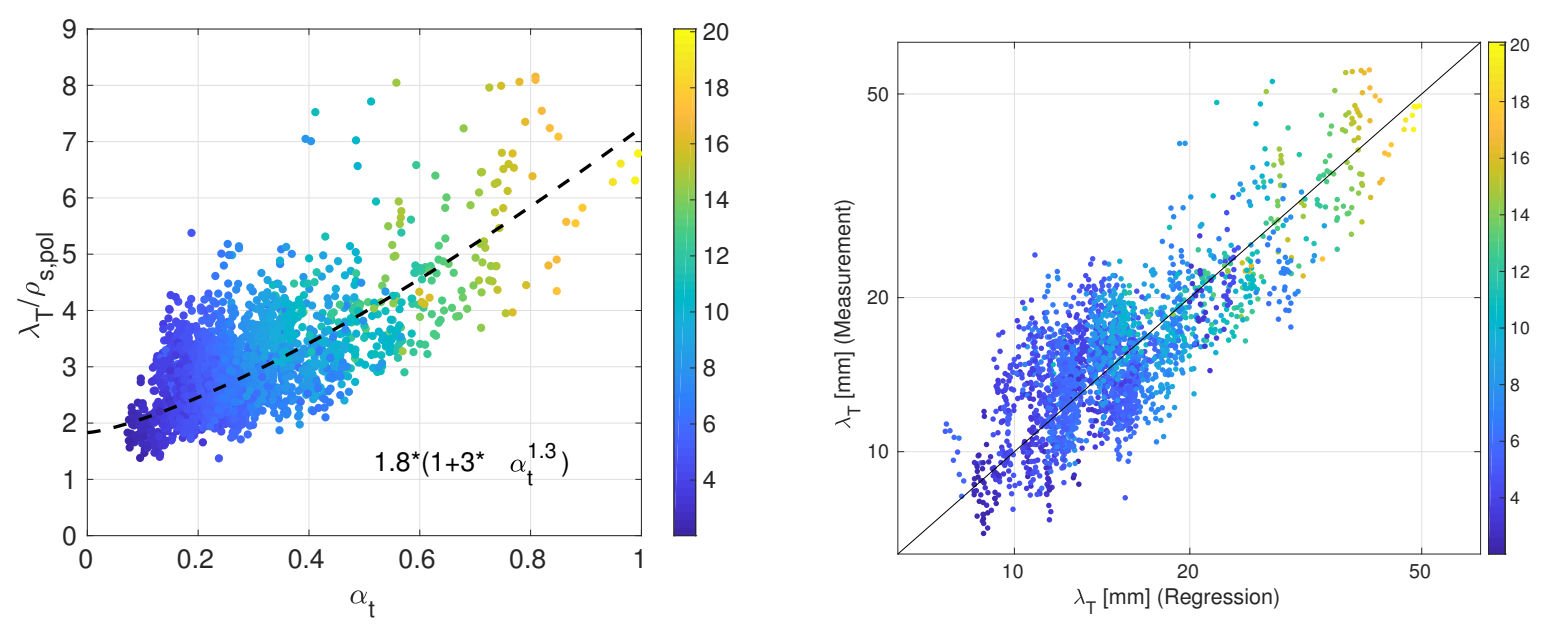

FIG. 8: (Left) Density decay length vs $\alpha_{\mathrm{t}}$. The dotted line show the turbulent broadening term of Eq. 22. (Right) Measurements versus regression values from Eq. 22. Color coding represents the separatrix collisionality (see Eq. 15)

density [43]). These conditions restrict the values for the turbulence parameter typically to $\alpha_{t} \simeq 0.2\left(n_{\mathrm{sep}} \simeq 2 \cdot 10^{-19} m^{-3}, T_{\mathrm{e}} \simeq 100 \mathrm{eV}\right.$ and $\left.\rho_{\mathrm{s}, \mathrm{pol}}=\sqrt{m_{\mathrm{D}} T_{\mathrm{e}}} /\left(e B_{\mathrm{pol}}\right)\right)$. Inserting $\alpha_{\mathrm{t}}$ into Eq.(22) gives

$$
\lambda_{\mathrm{T}}[\mathrm{mm}]=\left(1+3 \cdot 0.2^{1.3}\right) \cdot 1.8 \rho_{\mathrm{s}, \mathrm{pol}}=\frac{3.56}{B_{\mathrm{pol}}}
$$

By recalling that for ASDEX Upgrade geometry (see Eq.(9) from [7]) $\lambda_{\mathrm{q}, \mathrm{MP}} \simeq 0.55 \lambda_{\mathrm{q}}$ and $B_{\mathrm{pol}, \mathrm{MP}} \simeq 1.31 B_{\mathrm{pol}}$ it is

$$
\lambda_{\mathrm{q}, \mathrm{MP}}[\mathrm{mm}]=3.56 \cdot \frac{2}{7} \cdot 0.55 \cdot 1.31 \cdot B_{\mathrm{pol}, \mathrm{MP}}^{-1}=0.73 \cdot B_{\mathrm{pol}, \mathrm{MP}}^{-1}
$$

which is well in line with the ITPA outer divertor target power width scaling and the Alcator C-Mod studies by Brunner at al. [42].

\section{COMPARISON OF $\alpha_{\mathrm{t}}$ VS THE CONFINEMENT FACTOR $\mathbf{H}_{98, y 2}$}

The power width is observed to increase with the turbulence parameter. High performance plasmas in future fusion experiments such as ITER will need to find plasma operational schemes that combine a power exhaust solution with sufficient plasma performance, i.e. confinement factor $\mathrm{H}_{98, y 2}$ of about one. It is hence of interest to compare this parameter with the turbulence parameter $\alpha_{\mathrm{t}}$. Figure 9 presents for the H-Mode data base the 

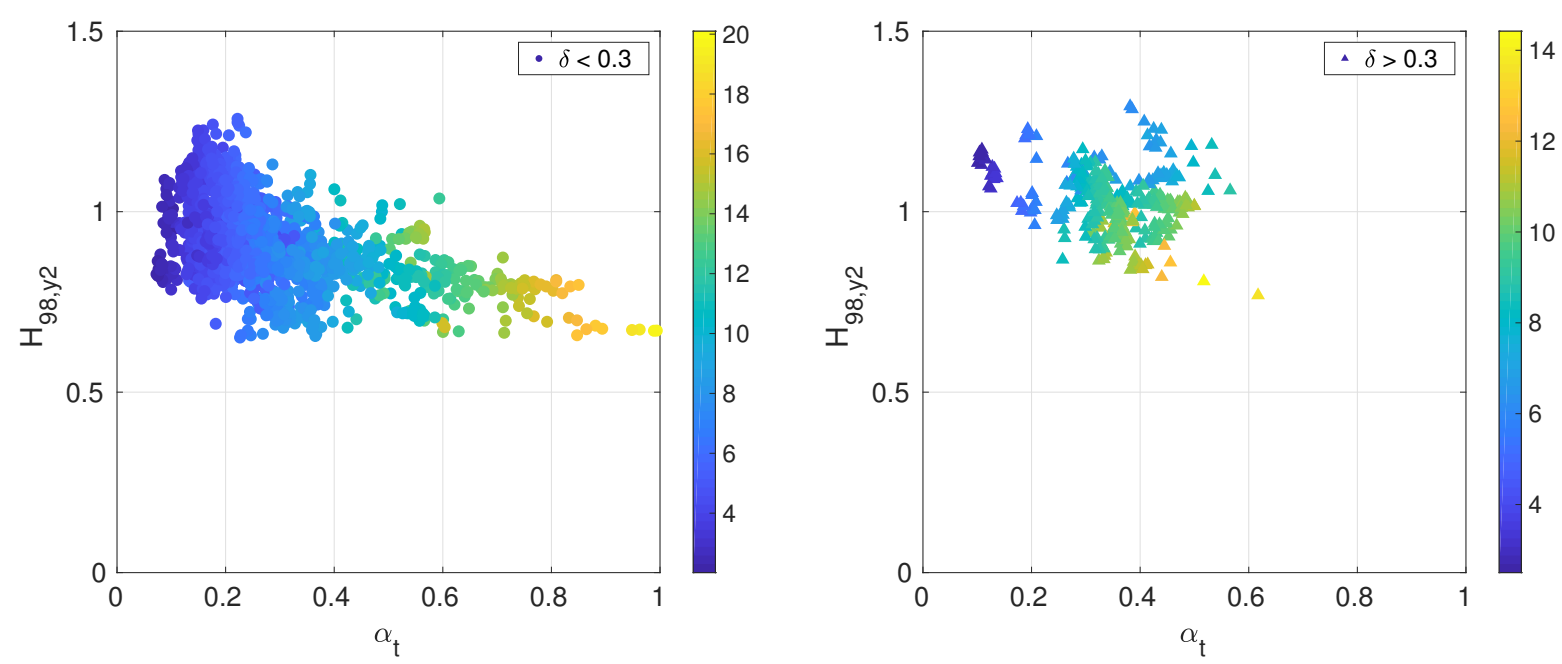

FIG. 9: The confinement factor $\mathrm{H}_{98 y, 2}$ is observed to lower when the turbulence parameter $\alpha_{\mathrm{t}}$ increases, here separated for low (left) and (right) high triangularity discharges. Higher values of $\mathrm{H}_{98 y, 2}$ are measured at a constant $\alpha_{\mathrm{t}}$ with increasing triangularity (at $\left.\rho_{\text {pol }}=0.95\right)$. Color coding represents the edge collisionality (see Eq. 15)

confinement factor versus $\alpha_{\mathrm{t}}$. In general confinement depends much on core and pedestal performance and a comparison with a local value like $\alpha_{\mathrm{t}}$ calculated at the separatrix is of limited explanatory power. Thus, the scatter is large.

As displayed in Fig. 9 (left), clearly the confinement factor lowers with increasing values for $\alpha_{\mathrm{t}}$. Further we see (right) that plasmas with higher triangularity, here represented by the magnetic equilibrium triangularity at $\rho_{\text {pol }}=0.95$, reach the highest confinement factor for a given value of the turbulence parameter, well recognizable for $0.1 \leq \alpha_{\mathrm{t}} \leq 0.5$. This has the important implication that a widening of the power width appears to be in general linked to a confinement reduction. The latter observation is well in line with the work by Brunner showing for C-Mod plasmas that the volume averaged pressure and the power width is correlated. Dedicated studies for AUG to validate the proposed scaling from Brunner [42], also by including L and I-Mode plasmas, will be published in a separated paper [44].

\section{SUMMARY}

In summary a generalization of the multi-machine H-Mode power width scaling is proposed by using solely local separatrix values. The choice from the variety of possibilities 
is simple, we stick to the poloidal ion sound Larmor gyro-radius as excellent agreement is reported from basically all operating tokamaks when using low density target measurements. As the new aspect we introduce the dimensionless parameter $\alpha_{\mathrm{t}}\left(\propto \alpha_{\mathrm{d}}^{-2}\right)$ which is proposed as a turbulence control parameter. This control parameter itself is machine size dependent, and hence inter-machine comparisons at high collisionality are of great interest for future collaborative experiments. Figure 6 (left) presents the widening effect of $\lambda_{\mathrm{p}} / \rho_{s, p o l}$ and here we point on two highly notable aspects. First, the ratio between $\lambda_{\mathrm{p}}$ and the poloidal ion sound Larmor gyro-radius is approaching unity when the turbulence parameter is approaching zero. Secondly, H-Mode data only exist for $\alpha_{\mathrm{t}} \leq 1$. When approaching $\alpha_{\mathrm{t}} \simeq 1$ a significant widening of pressure fall-off length is observed while direct measurements from DBS show an increased level of turbulence and a reduction of the confinement factor until L-Mode conditions are hit. The strength of the increasing turbulence parameter is different for the temperature and density decay lengths. Whereas the density channel appears to widen more strongly, a more moderate widening of the electron temperature fall-off length, or the power width, is observed. A significant widening $(\simeq 2)$ is only observed at values of $\alpha_{\mathrm{t}} \geq 0.5$ for which the H-Mode data base for ASDEX Upgrade displays a reduction of the confinement factor $H_{98, y 2}$.

Future work will compare the new data base with available models presented in the introduction section potentially allowing for a separation of effects on the perpendicular drift-ordered and parallel SOL transport. A generalization of the Heuristic Drift model [45] to finite collisonality could also act synergistically with the here proposed direct turbulence enhancement. The influence of $\alpha_{\mathrm{t}} \rightarrow 1$ on the pedestal performance i.e. for high triangularity plasmas, is of great interest for future high performance devices such as ITER.

\section{ACKNOWLEDGEMENT}

This work has been carried out within the framework of the EUROfusion Consortium and has received funding from the Euratom research and training programme 2014-2018 and 2019-2020 under grant agreement No 633053. The views and opinions expressed herein do not necessarily reflect those of the European Commission. The work of RJG was supported by US contract No.DE-AC02-09CH11466. 


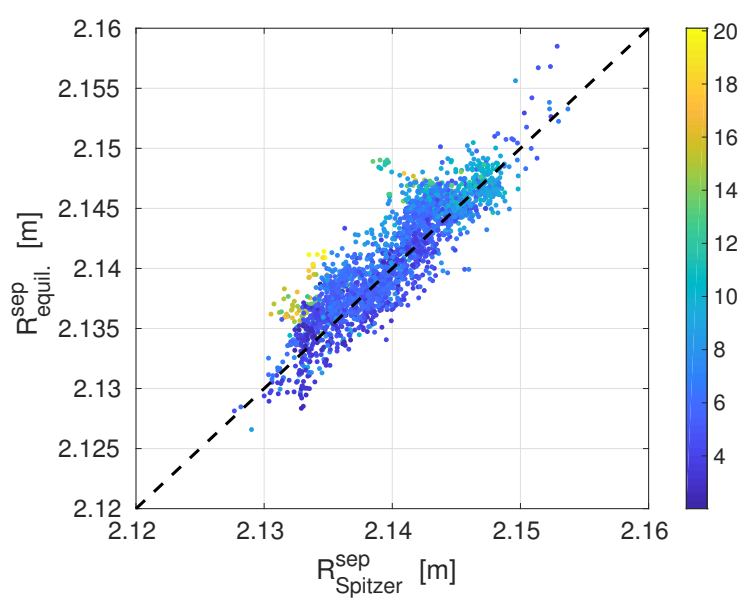

FIG. 10: Estimated separatrix position by solving Eq. A1 versus the separatrix position from magnetic equilibrium estimation. The mean difference is estimated to be $1.1 \mathrm{~mm}$. Color coding represents the edge collisionality (see Eq. 15)

\section{Appendix A: Validation of separatrix position and decay length estimation}

To derive the separatrix density and temperature and their (poloidally averaged) falloff lengths we use basically the same method as in [15] and [16] though with some minor differences as described next. These differences are driven by our ambition to establish a very large data base and hence needs to be processed automatically. Each discharge is divided into 20 time windows. Start and end point of the discharges are taken as the flat top phase for the plasma current with a minimum length of 2 seconds. Each time window has as a length between $250 \mathrm{~ms}$ and about $500 \mathrm{~ms}$ depending on the discharge flat top duration. Type-I ELMs are taken out from the analysis, however small ELMs or larger ballooning features cannot be excluded from the data analysis. Distinction between L and H-Mode intervals are done by hand for each discharge. We apply the model by Stangeby [46] to estimate the electron temperature, $T_{e, s e p}$ starting with $\lambda_{\mathrm{q}}$ from the multi-machine scaling:

$$
T_{e, s e p} \approx\left(\frac{7 P_{s e p} \hat{q}_{c y l}^{2} A}{16 \kappa_{0}^{e} \hat{\kappa} \lambda_{\mathrm{q}}}\right)^{\frac{2}{7}} \quad \text { with } \quad \lambda_{\mathrm{q}}=\frac{2}{7} \lambda_{\mathrm{T}}
$$

A simple iterative numerical scheme then successively solves Eq. A1 by searching for the position in the electron temperature profiles from Thomson-Scattering data where Eq. (A1) is fulfilled such that the calculated separatrix electron temperature and the measured one agree within $2 \%$. This method works well for about $85 \%$ of the analyzed time intervals. For 
the remaining $15 \%$ of the time windows no data are generated. Typically these time intervals are when the magnetic equilibrium is changing due to $\mathrm{L}-\mathrm{H}$ transitions or changes in plasma positioning and shaping. Figure 10 presents the estimated separatrix position for H-Mode data points from the iterative method and the magnetic reconstruction. The mean difference between both methods is only $1.1 \mathrm{~mm}$ and the standard deviation is $2.2 \mathrm{~mm}$. However, we note that the mean difference for data with high collisionality is somewhat more pronounced. It is currently unclear to which extent this is due to the less well fulfilled assumptions of the Stangeby model or magnetic reconstruction. The expression for the parallel heat constant is taken from [5] and writes as

$$
\begin{aligned}
\kappa_{0}^{e} & =\frac{2600}{f_{\kappa, 0}\left(Z_{\text {eff }}\right)} \frac{\mathrm{W}}{(\mathrm{eV})^{\frac{7}{2}} \mathrm{~m}} \text { with } \\
f_{\kappa, 0}\left(Z_{\mathrm{eff}}\right) & =0.672+0.076 Z_{\mathrm{eff}}^{0.5}+0.252 Z_{\mathrm{eff}}
\end{aligned}
$$

\section{Appendix B: Dependence of $\alpha_{\mathrm{t}}$ on $Z_{\mathrm{eff}}$}

The characteristic ion mass $M_{i}$ in a multi-species plasma should obey quasi-neutrality $n_{e} M_{i}=\sum_{j} n_{j} M_{j}$, where $M_{j}$ and $n_{j}$ are mass and density of the different ion species, respectively. Defining an average ion mass $\bar{M}=\left(\sum_{j} n_{j} M_{j}\right) /\left(\sum_{j} n_{j}\right)$ the characteristic ion mass is given by $M_{i}=\bar{M} / \bar{Z}$. For single charge ions the characteristic electron-ion collision frequency is similar to that of electron-electron collisions $\nu_{\mathrm{ei}} \approx \nu_{\mathrm{ee}}$ and hence often both are used equivalently. The electron-electron Braginskii collision frequency is given by

$$
\nu_{\mathrm{ee}}=\frac{4 \sqrt{2 \pi}}{3} \frac{n_{\mathrm{e}} e^{4} \ln \Lambda}{\left(4 \pi \epsilon_{0}\right)^{2} \sqrt{m_{\mathrm{e}}} T_{\mathrm{e}}^{3 / 2}} .
$$

The term $0.51 \nu_{\mathrm{ei}}$ should describe the electric resistivity, which is only valid for single charge ions. To take into account multiple ion species with finite $Z_{\text {eff }}$ defined by $n_{\mathrm{e}} Z_{\text {eff }}=\sum_{j} n_{j} Z_{j}^{2}$, the Braginskii result is fitted for $Z_{\text {eff }}=1,2,3,4$ and $\infty$. This yields

$$
\nu_{e i}=Z_{\text {eff }} f_{\mathrm{Z}_{\mathrm{eff}}} \nu_{\mathrm{ei}}\left(Z_{\mathrm{eff}}=1\right) \simeq Z_{\mathrm{eff}} f_{\mathrm{Z}_{\mathrm{eff}}} \nu_{\mathrm{ee}}
$$

where $f_{\mathrm{Z}_{\text {eff }}}$ is defined as

$$
f_{\mathrm{Z}_{\mathrm{eff}}}=(1-0.569) \exp \left(-\left(\frac{\left(Z_{\mathrm{eff}}-1\right)}{3.25}\right)^{0.85}\right)+0.569 .
$$


The latter function varies only slightly for $Z_{\text {eff }}$, for typical values of $Z_{\text {eff }}=1.25$ for unseeded and clean plasmas we estimate $f_{\mathrm{Z}_{\mathrm{eff}}}=0.95$ with boron as the main impurity. Even for plasmas with high content of impurities $Z_{\text {eff }}=2.5$ the value for $f_{\mathrm{Z}_{\mathrm{eff}}}$ is 0.83 . The mean value of the data base is $Z_{\text {eff }}=1.33$ and $f_{\mathrm{Z}_{\text {eff }}}=0.94$, reflecting that mainly unseeded clean plasmas are investigated. Also we added $1 / \bar{Z}$ in $\omega_{\mathrm{B}}$ (see Eq. 7) to take into account the dilution by impurities to the ion pressure to the interchange forcing.

[1] F.Wagner, Phys. Rev. Lett. 49. p.1408 (1982)

[2] H.Zohm, Nucl. Fusion, p.073019 Vol.53 (2013)

[3] A.S. Kukushkin, H.D. Pacher, G.W. Pacher et al., J. Nucl. Mater., p.S203-207, Vol. 438 (2013)

[4] M.L.Reinke, Nucl. Fusion 57, p. 034004 (2017)

[5] R.J.Goldston, M.L.Reinke, J.A.Schwartz, Plasma Phys. Contr. Fusion, p.055015 Vol.59 (2017)

[6] A.Kallenbach et al., p. 166-174, Vol.18, Nuclear Materials and Energy

[7] T.Eich, B.Sieglin et al., Phys. Rev. Lett. 195003 (2011)

[8] T.Eich, R.A.Pitts, A.W.Leonard et al., Nucl. Fusion, p.093031 Vol.53 (2013)

[9] R.J.Goldston, Nucl. Fusion, p.013009 Vol.52 (2012)

[10] V.Rozhansky, E.Kaveeva, I.Senichenkov et al., Plasma Phys. Contr. Fusion 60, p.035001 (2018)

[11] R.J.Goldston, J. Nucl. Mater., p.397-400, Vol.463 (2015)

[12] C.S.Chang, S.H.Ku, A.Loarte et al., Nucl.Fusion, p.116023, Vol.57 (2017)

[13] I.Keramidas Charidakos, J.R.Myra, S.Parker et al., Physics of Plasmas 25, 072306 (2018)

[14] M.Bernert,T.Eich, A.Kallenbach et al., Plasma Phys. Contr. Fusion, p.014038 Vol.57 (2015)

[15] H.J Sun, E.Wolfrum ,T.Eich et al., Plasma Phys. Contr. Fusion, p.125001 Vol.57 (2015)

[16] T.Eich, R.J.Goldston, A.Kallenbach et al., Nucl. Fusion, p.034001 Vol.58 (2018)

[17] M.Faitsch, B.Sieglin, T.Eich et al. Plasma Phys. Contr. Fusion, p.075005 Vol.57 (2015)

[18] M.A.Makowski, C.J.Lasnier, A.W.Leonard et al., Nuclear Materials and Energy, p.1010-1014, Vol.12 (2017)

[19] A.W.Leonard, A.G.McLean, M.A.Makowski and P.C.Stangeby, Nucl. Fusion, p.086033 Vol.57 (2017)

[20] M.Greenwald, Plasma Phys.Contr.Fusion,R27, 44 (2002)

[21] J.Myra, D.A.D'Ippolito and D.A.Russel, Physics of Plasmas 22, 042516 (2015) 
[22] F.Halpern, S.Jolliet, J.Loizu et al., Physiscs of Plasmas, Vol. 20, 052306 (2013)

[23] F.Militello, V.Naulin and A.H.Nielsen, Plasma Phys. Contr. Fusion 55, 074010 (2013)

[24] J.Olsen, A.H.Nielsen, J.J.Rasmussen et al., Plasma Phys. Contr. Fusion 60, 085018 (2018)

[25] B.Chen, X.Q.Xu, T.Y.Xia et al., Physics of Plasmas 25, 055905 (2018)

[26] N.Fedorczak, M.Peret, H.Bufferand et al., Nuclear Materials and Energy, p.433-439, Vol.19 (2019)

[27] B.N.Rogers, J.F.Drake, A.Zeiler, Phys. Rev. Lett. Vol.81, p.4396 (1998)

[28] B.D. Scott, Physics of Plasmas 12, 062314 (2005)

[29] B.D. Scott, Plasma Phys. Contr. Fusion 49, S25 (2007)

[30] W.Suttrop, V.Mertens, H.Murmann et al., J. Nucl. Mater., p.118, Vol.266-269 (1999)

[31] B.LaBombard, J.W.Hughes, D.Mossessian et al., Nucl. Fusion, p.1658 Vol.45 (2005)

[32] B.D. Scott, New Journal Physics 4, 52 (2002)

[33] R.Hong et al., Nucl. Fusion 58, 016041 (2018)

[34] R.J. Haijar, P.H. Diamond, M.A. Malkov, Phys. Plasmas 25, 062306 (2018)

[35] B. LaBombard, R. L. Boivin, M. Greenwald et al., Phys. Plasmas 8, p.2107 (2001).

[36] G.Y.Antar, G.Counsel, and J.W.Ahn, Physics of Plasmas 12, 082503 (2008)

[37] J. Pinzon , T. Happel , P. Hennequin et al., Nuclear Fusion, Vol.59 074002 (2019)

[38] P.Hennequin et al., Contributions to 44th EPS Conference on Plasma Physics, Belfast, Ireland (2017)

[39] T.Happel, T.Görler, P.Hennequin et al., Plasma Phys. Contr. Fusion, p.054009 Vol.59 (2017)

[40] H.J Sun, E.Wolfrum ,B.Kurzan et al., Plasma Phys. Contr. Fusion, p.105010 Vol.59 (2017)

[41] H.J Sun, E.Wolfrum ,T.Eich et al., Plasma Phys. Contr. Fusion, p.014005 Vol.61 (2019)

[42] D.Brunner, B.LaBombard, A.Q.Kuang, J.L. Terry, Nuclear Fusion, Vol.58 094002 (2018)

[43] A.Kallenbach, H.J.Sun, T.Eich et al., Plasma Phys. Contr. Fusion, p.045006 Vol.60 (2018)

[44] D.Silvagni, T.Eich, M.Faitsch et al., submitted to Plasma Phys. Contr. Fusion

[45] R.J.Goldston, T.Eich, Contribution to EPS-44, Milan, Italy (2019)

[46] P.C.Stangeby, The plasma boundary of magnetic fusion devices, Inst.of Phys.Publishing (2000) 